\title{
Tuning the Mott Transition in a Bose-Einstein Condensate by Multiple Photon Absorption
}

\author{
C. E. Creffield and T. S. Monteiro \\ Department of Physics and Astronomy, University College London, Gower Street, London WC1E 6BT, United Kingdom
}

(Received 4 April 2006; published 1 June 2006)

\begin{abstract}
We study the time-dependent dynamics of a Bose-Einstein condensate trapped in an optical lattice. Modeling the system as a Bose-Hubbard model, we show how applying a periodic driving field can induce coherent destruction of tunneling. In the low-frequency regime, we obtain the novel result that the destruction of tunneling displays extremely sharp peaks when the driving frequency is resonant with the depth of the trapping potential ("multi-photon resonances"), which allows the quantum phase transition between the Mott insulator and the superfluid state to be controlled with high precision. We further show how the waveform of the field can be chosen to maximize this effect.
\end{abstract}

DOI: 10.1103/PhysRevLett.96.210403

Recent spectacular progress in trapping cold atomic gases [1] has provided a new arena for studying quantum many-body physics. In particular, ultracold bosons held in optical potentials provide an almost ideal realization of the Bose-Hubbard (BH) model [2], in which the model parameters can be controlled to high precision. As well as their purely theoretical interest, these systems attract attention because of their possible application to quantum information processing [3].

The $\mathrm{BH}$ model is described by the Hamiltonian

$$
H_{\mathrm{BH}}=-J \sum_{\langle i, j\rangle}\left[a_{i}^{\dagger} a_{j}+\text { H.c. }\right]+\frac{U}{2} \sum_{i} n_{i}\left(n_{i}-1\right),
$$

where $a_{i}\left(a_{i}^{\dagger}\right)$ are the standard annihilation (creation) operators for a boson on site $i, n_{i}=a_{i}^{\dagger} a_{i}$ is the number operator, $J$ is the tunneling amplitude between neighboring sites, and $U$ is the repulsion between a pair of bosons occupying the same site. Its physics is governed by the competition between the kinetic energy and the Hubbard interaction, and thus by the ratio $U / J$. When $U / J \ll 1$ the tunneling dominates, and the ground state of the system is a superfluid. As $U / J$ is increased the system passes through a quantum phase transition, and evolves into a Mottinsulator (MI) state in which the bosons localize on the lattice sites.

This phase transition was observed experimentally in Ref. [4] by varying the depth of the optical potential. In this Letter we propose an alternative method: applying an additional oscillatory potential induces coherent destruction of tunneling (CDT), and thus suppresses the effect of $J$. CDT is a quantum interference effect, discovered in the pioneering work of Ref. [5], in which the period for tunneling between states diverges as their associated quasienergies [6] approach degeneracy.

Here we show how CDT can be used to control the dynamics of a boson condensate, by means of a novel resonance effect between $U$ and the frequency of the driving field. We consider a one-dimensional BH model, driven by a time-periodic potential which varies linearly
PACS numbers: 05.30.Jp, 03.65.Xp, 03.75.Lm, 73.43.Nq

with site number. The Hamiltonian is given by

$$
H(t)=H_{\mathrm{BH}}+K f(t) \sum_{j}^{N} j n_{j},
$$

where $K$ is the amplitude of the driving field, and $f(t)$ is a $T$-periodic function of unit amplitude that describes its waveform. Such time-periodic linear potentials - generated by an accelerated lattice for example - have already been used in cold-atom experiments [7]. A similar form of driving potential was also recently investigated theoretically [8] in the high-frequency regime $(\omega>U)$, and was found to suppress the transition to the superfluid regime.

Here, for the first time, the multiphoton (low-frequency) regime is investigated. An unexpected new finding is that CDT is now modulated by a set of extremely sharp "resonances" [the contrast between the high-frequency behavior and the multiphoton regime investigated here is illustrated in Fig. 1(a)]. This means that the Mott transition can be induced by minute changes in experimental parameters.

Henceforth we put $\hbar=1$ and measure all energies in units of $J$, and set the number of bosons equal to the number of lattice sites $N$. Although the dimension of the Hilbert space increases exponentially with $N$, in a Fock basis $H$ is extremely sparse, with at most $(2 N-1)$ nonzero entries per row. Thus despite the rapid increase in the dimension of the Hilbert space, this sparsity allows us to treat relatively large systems of up to 11 sites, and so assess if the effects we observe survive in the thermodynamic limit.

Our numerical investigation consists of initializing the system in the "ideal" MI state, $\left|\Psi_{\text {MI }}\right\rangle=\prod a_{j}^{\dagger}|0\rangle$, and evolving the many-particle Schrödinger equation over time (typically ten periods of the driving field) using a Runge-Kutta method. To study the system's time-evolution quantitatively, we measure the overlap of the wave function with the initial state $P(t)=\left|\left\langle\Psi_{\mathrm{MI}} \mid \Psi(t)\right\rangle\right|^{2}$. For convenience we term the minimum value of $P(t)$ attained during the time evolution to be the localization. When CDT occurs, the system will remain frozen in the MI state, 


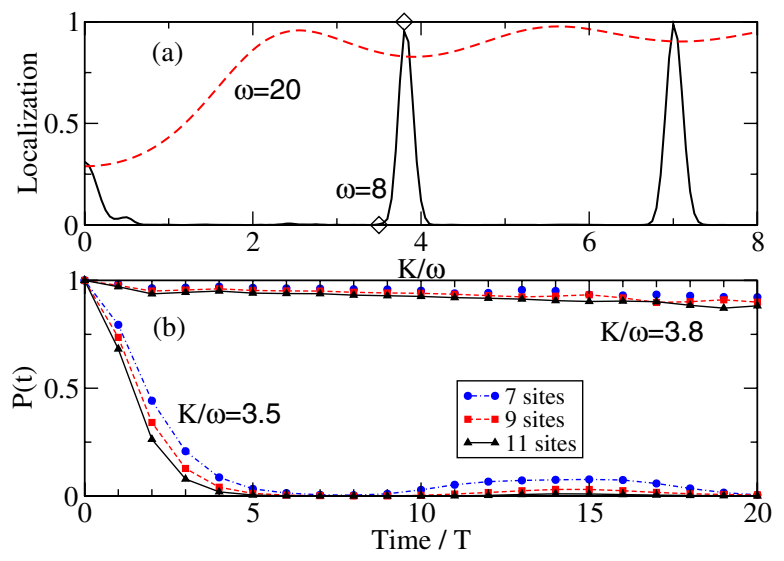

FIG. 1 (color online). (a) The minimum overlap with the MI state, or localization, reached in a 7-site system with $U=8$, during 10 periods of driving. For $\omega=20$ (dashed line) the localization peaks at $K / \omega=2.4,5.5$ - the zeros of $\mathcal{J}_{0}(K / \omega)$. When $\omega$ is reduced to $\omega=8$ (the first photon resonance, solid line), the peaks become extremely narrow and are centered on the zeros of $\mathcal{J}_{1}(K / \omega)$. The diamonds mark the points $K / \omega=$ 3.5 and 3.8 (see below). (b) Time evolution of the $\omega=8$ case for three system sizes, 7, 9 , and 11 sites. For $K / \omega=3.5$, away from the resonance, the overlap with the initial state, $P(t)$, rapidly drops to zero. For $K / \omega=3.8$, at the peak of the resonance, the decay is much slower, indicating that the driving field preserves the MI state.

and consequently the localization will be close to 1 . Conversely, if the bosons are able to tunnel freely from site to site, the value of the localization will be reduced.

We begin by considering the case of sinusoidal driving, $f(t)=\sin \omega t$. The MI transition is quite soft in $1 \mathrm{D}$, starting at $U \simeq 4$ and developing fully for $U>20$. Throughout this work we use an intermediate value of $U=8$. Figure 1(a) shows how the localization in a 7-site system varies as the amplitude of the driving field is increased, while its frequency is held constant at $\omega=20$. For $K=0$ the localization has a value of $\sim 0.3$, demonstrating that in the absence of a driving field this value of $U$ is indeed insufficient to maintain the MI state. Applying the driving field causes the localization to steadily rise from this value as $K$ is increased from zero, indicating that the effective tunneling between lattice sites is increasingly suppressed, until it peaks at a value close to 1 at $K / \omega=2.4$. As $K$ is increased further, the localization goes through a shallow local minimum, before again peaking at $K / \omega=5.5$. It was observed [8] that these values of $K / \omega$ are close to the first two zeros of $\mathcal{J}_{0}$, the zeroth Bessel function.

Reducing the driving frequency to a lower value, $\omega=8$, produces a radically different behavior - the value of the localization rapidly drops as $K / \omega$ is increased from zero, indicating that the field destroys the MI state. As $K / \omega$ is increased further the value of the localization remains extremely low except at a series of very sharp peaks. Figure 1(b) emphasizes the narrowness of these peaks by showing the time evolution of the system for two values of
$K$. For the first, $K=3.5 \omega, P(t)$ rapidly falls from its initial value, reaching a level near zero within five driving periods. There is a small dependence on the system size, with the decay occurring more quickly as $N$ is increased. At the localization peak, $K=3.8 \omega, P(t)$ decays far more slowly with time, so that after 20 periods of driving it only falls to a value of $\sim 0.9$, and only minor dependence on $N$ is evident. Thus for this value of $\omega$, altering the amplitude of the field by just $10 \%$ produces enormous differences in the localization.

Although the Hamiltonian (2) is explicitly time dependent, the fact that it is periodic allows us to use the Floquet theorem to write solutions of the Schrödinger equation as $\psi(t)=\exp \left[-i \epsilon_{j} t\right] \phi_{j}(t)$, where $\epsilon_{j}$ is the quasienergy, and $\phi_{j}(t)$ is a $T$-periodic function called the Floquet state [6]. As the quasienergies are only defined up to an arbitrary multiple of $\omega$, the quasienergy spectrum possesses a Brillouin zone structure, in precise analogy to the quasimomentum in spatially periodic crystals. For the Floquet analysis, we work in an extended Hilbert space of $T$-periodic functions [9]. In this approach, the Floquet states and quasienergies satisfy

$$
\mathcal{H}(t)\left|\phi_{j}(t)\right\rangle=\epsilon_{j}\left|\phi_{j}(t)\right\rangle,
$$

where $\mathcal{H}(t)=H(t)-i \hbar \partial / \partial t$. Working in this extended Hilbert space thus reduces the task of calculating the timedependent, driven dynamics of the system to a time-independent eigenvalue problem.

To study the behavior of the quasienergies, we make use of a perturbative scheme developed in Ref. [10] to treat noninteracting systems, and later generalized in Ref. [11] to include interactions. Our procedure is to first find the eigensystem of the operator $\mathcal{H}_{0}(t)=H_{0}(t)-i \hbar \partial / \partial t$, where $H_{0}$ contains terms diagonal in a Fock basis (i.e., the driving term and the Hubbard interaction). We are then able to use standard Rayleigh-Schrödinger perturbation theory to evaluate the corrections to this result, using the remaining terms of $H_{\mathrm{BH}}$ as the perturbation.

For the two-site system, a natural basis is given by the Fock states $\{|1,1\rangle,|2,0\rangle,|0,2\rangle\}$, where $|n, m\rangle$ denotes the state with $n$ bosons on the first site and $m$ on the second. Finding the eigensystem of $\mathcal{H}_{0}$ then amounts to solving three first-order differential equations, yielding the result

$$
\begin{aligned}
\left|\phi_{+}(t)\right\rangle & =\left(0, \exp \left[-i\left(U-\epsilon_{+}\right) t+i \frac{K}{\omega} \cos \omega t\right], 0\right) \\
\left|\phi_{0}(t)\right\rangle & =\left(0,0, \exp \left[-i\left(U-\epsilon_{0}\right) t-i \frac{K}{\omega} \cos \omega t\right]\right) \\
\left|\phi_{-}(t)\right\rangle & =\left(\exp \left[i \epsilon_{-} t\right], 0,0\right) .
\end{aligned}
$$

Imposing the $T$-periodic boundary condition on these states requires setting $\epsilon_{-}=0$ and $\left(U-\epsilon_{+/ 0}\right)=m \omega$, where $m$ is an integer. Thus in general it is not possible to include the full Hubbard-interaction term within $H_{0}$, depending on its commensurability with $\omega$. To deal with this it is necessary to decompose $U$ into a form which 
duplicates the Brillouin zone structure of the quasienergies

$$
U=n \omega+u, \quad n=0,1,2 \ldots
$$

where $u$ is the "reduced interaction," $|u| \leq \omega / 2$. This decomposition reveals that only the reduced interaction needs to be included in the perturbation, while the remainder of $U$ (an integer multiple of $\omega$ ) can be retained in $H_{0}$.

To first order it is easily shown that the three quasienergies are given by

$$
\epsilon_{0}=u \quad \text { and } \quad \epsilon_{ \pm}=\left(u \pm \sqrt{u^{2}+16 J_{\text {eff }}^{2}}\right) / 2,
$$

where the intersite tunneling has been reduced to an effective value $J_{\text {eff }}=J \mathcal{J}_{n}(K / \omega)$, and $n$ and $u$ are defined in Eq. (5). Thus in the high-frequency limit $(\omega \gg U)$, when $n=0$ and $u=U$, it is clear that the quasienergies $\epsilon_{0}$ and $\epsilon_{+}$are degenerate when $\mathcal{J}_{0}(K / \omega)=0$. In Fig. 2(a) we show the excellent agreement between the perturbative result and the exact quasienergies for a driving field of frequency $\omega=20$. In Fig. 2(b) we plot the corresponding value of the localization, and it can be clearly seen that the peaks in this quantity are indeed centered on the points of closest approach of the quasienergies. It may be seen from Eq. (6) that for large values of $u$, the quasienergy separation $\left(\epsilon_{+}-\epsilon_{0}\right) \simeq 4 J_{\text {eff }}^{2} / u$. The effect of $u$ is thus to reduce the amplitude of oscillations in this quantity, and so to smear out the avoided crossings of the quasienergies. As a result, the peaks in the localization are rather broad and

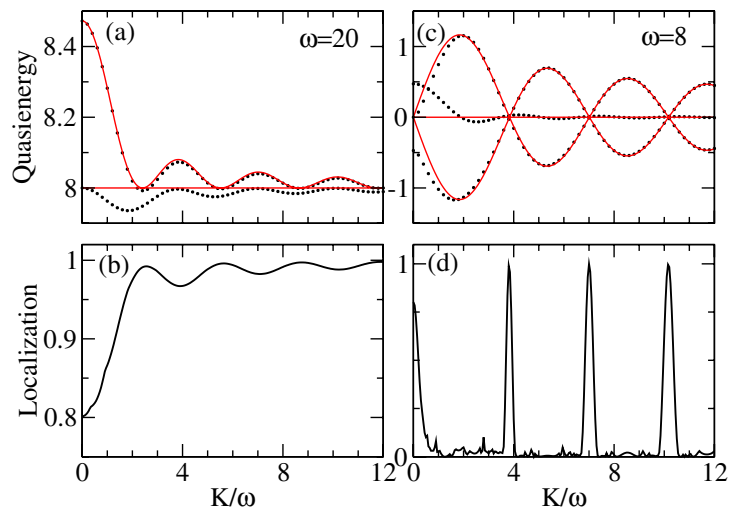

FIG. 2 (color online). (a) Quasienergy spectrum of a 2-site system, with $U=8$ and $\omega=20$. Only two of the three quasienergies are plotted (the remaining one oscillates weakly about zero), which make a series of close approaches to each other as $K$ is increased. Solid lines in (a) and (c) (red online) denote the perturbative solutions, which agree well with the exact results (black circles). (b) At the points of close approach, the tunneling is suppressed and the localization peaks. For all field strengths the tunneling is suppressed with respect to the undriven system, and the localization is thus enhanced. (c) At lower frequencies the behavior of the quasienergies changes dramatically. At $\omega=8$ the system is at the first photon resonance ( $U=$ $\omega$ ), and the behavior of the quasienergies is described extremely well by the perturbative solutions $\epsilon=0$, $\pm 2 \mathcal{J}_{1}(K / \omega)$. (d) As before, the localization is peaked at the points of quasienergy crossing, but in contrast to (b) the peaks are extremely sharp. overlap each other, and thus the localization cannot reach particularly low values.

Equation (5) reveals the particular importance of photon resonances, when $U$ is an integer multiple of the frequency of the driving field, $U=n \omega$. When this condition is satisfied the reduced interaction is zero, and the crossings between the quasienergies are well-defined. This is the origin of the extremely sharp peaks in localization seen in Fig. 1(a) for $\omega=U=8$. Away from these peaks, the photon absorption compensates for the energy cost of doubly occupying a lattice site, in analogy to the photonassisted tunneling studied in Ref. [12], thereby producing low values of localization. In Fig. 2(c) we plot the quasienergies for the first photon resonance $(n=1)$ for the twosite system. For weak fields $(K / \omega<2)$ small deviations of the exact quasienergies from the perturbative result are visible, but for higher field strengths the agreement is again excellent. In Fig. 2(d) we plot the localization produced in this system, and we can note that, as seen previously in the 7 -site system, the localization takes extremely low values except at a set of very narrow peaks. These peaks are precisely aligned with the quasienergy crossings at the zeros of $\mathcal{J}_{1}(K / \omega)$.

As we reduce $\omega$ still further, we can expect to encounter a sequence of higher resonances with similar behavior. In Fig. 3(a) we compare the values of localization produced in a 7-site system for the $n=1$ and $n=2$ resonances. The second photon resonance, however, produces a worse result than for $n=1$. Although sharp peaks are still present in the localization, and in agreement with the perturbation theory they are indeed centered on the zeros of $\mathcal{J}_{2}(K / \omega)$, the maximum value of localization produced is considerably lower.
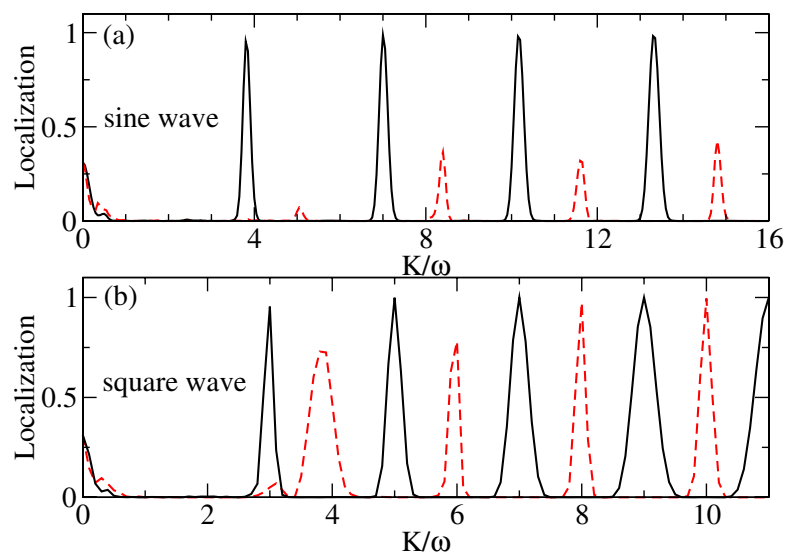

FIG. 3 (color online). Localization produced in a 7-site system $(U=8)$ for two forms of periodic driving: (a) sinusoidal, (b) square wave. Both waveforms produce excellent localization at the first photon resonance, $\omega=U$, shown by solid black lines. At the second photon resonance $(2 \omega=U)$, shown by dashed lines (red online), the localization produced by the sinusoidal driving is considerably smaller, but the square wave still produces high peaks. 
This poor localization occurs because not all of the quasienergy crossings in the Floquet spectrum of a many-site system will occur at precisely the same value of $K / \omega$; instead the various crossings occur over an interval. Thus although at the peaks in the localization many pairs of Floquet states will be degenerate (and tunneling between them will be suppressed), other state-pairs will only be approximately degenerate and will permit a small, but nonzero, degree of tunneling to occur. The major factors influencing this effect arise from higher-order terms in the expansion of the single-period time-evolution operator $U(T, 0)$, which manifest as multiparticle tunneling and tunneling beyond nearest neighbors. CDT is a quantum interference effect, which occurs when the dynamical phase acquired by a particle in a period of driving produces destructive interference, thereby suppressing the particle's dynamics. If, however, a "clump" of $n_{1}$ bosons tunnels between sites, the dynamical phase will be $n_{1}$ times larger than that for a single boson. Similarly, if a boson tunnels between two sites separated by $n_{2}>1$ lattice spacings, the dynamical phase will be $n_{2}$ times larger. For sinusoidal driving, the single-particle tunneling is suppressed when $\mathcal{J}_{n}(K / \omega)=0$; for these higher-order processes also to be suppressed we therefore also require $\mathcal{J}_{n}\left(n_{1} n_{2} K / \omega\right)=0$ for integers $n_{1}, n_{2}=1,2 \ldots N$.

Clearly this condition cannot be satisfied for sinusoidal driving, as the zeros of $\mathcal{J}_{n}(x)$ are not equally spaced. Thus to observe good localization properties at high photon resonances we need to construct a driving field $f(t)$ such that the crossings in its Floquet spectrum are periodically spaced. This problem was confronted in a different context in Ref. [13], where it was shown that such a field must be discontinuous at changes of sign. Possibly the simplest field of this type, and the most convenient for experiment, is a square wave field.

In Fig. 3(b) we show the localization obtained in a 7-site system driven by a square wave. Using the same perturbative approach as before, it may be shown that the quasienergy degeneracies occur for $(K / \omega)=2 m+1$ or $2 m$, depending on whether the order of the resonance $n$ is odd or even. Unlike the case of sinusoidal driving, the $n=2$ resonance displays good localization, comparable to that obtained for $n=1$. A contour plot showing the localization as a function of both $K / \omega$ and $\omega^{-1}$ is presented in Fig. 4. The prominent horizontal bands correspond to the photon resonances $\left(\omega^{-1}=n / U\right)$, which are punctuated by a series of narrow peaks at which the localization is preserved. This plot also clearly shows the division between the fairly featureless, poorly localized, "weak-driving" regime to the upper left, and the "strong-driving" regime which shows the resonance features. For the latter, the dynamics of the system are dominated by the combined effect of the driving field and the Hubbard interaction, and thus is well described by our form of perturbation theory.

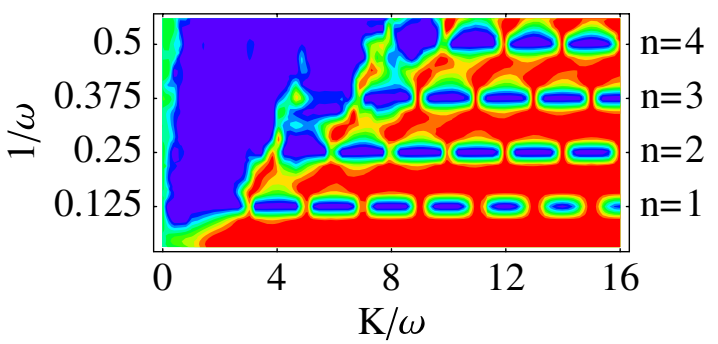

FIG. 4 (color online). The localization produced in a 5-site system with $U=8$ as a function of the frequency $\omega$ of the square wave driving field and its amplitude $K$. When $n \omega=U$ the localization is almost zero [the centers of the horizontal bands (blue online)] except at sharply defined peaks (red background online). Between the bands localization is good. The $n=$ $1,2,3$, and 4 resonances are marked on the right. The upper-left triangle (blue online) displays poor localization and little structure, corresponding to the nonperturbative regime.

Figure 4 allows us to locate the boundary between the two regimes quite accurately as $K / \omega \simeq(2 U) / \omega$.

In summary, we have investigated the dynamics of the BH model under a periodic driving field. For high frequencies [8] the field can be used to inhibit tunneling by means of CDT and thus stabilize the MI state. Lowering the frequency, however, reveals the existence of resonance effects which can be used to selectively destroy or preserve the MI state. Lower driving frequencies have the added advantages in experiment that they heat the condensate less, and will not drive transitions to higher Bloch bands thereby invalidating the single band model. The extremely narrow width of the resonance features indicates that it should be possible to control the Mott transition very precisely in this manner.

[1] O. Morsch and M. Oberthaler, Rev. Mod. Phys. 78, 179 (2006).

[2] D. Jaksch et al., Phys. Rev. Lett. 81, 3108 (1998).

[3] D. Jaksch et al., Phys. Rev. Lett. 82, 1975 (1999).

[4] M. Greiner et al., Nature (London) 415, 39 (2002).

[5] F. Grossmann, T. Dittrich, P. Jung, and P. Hänggi, Phys. Rev. Lett. 67, 516 (1991).

[6] M. Grifoni and P. Hänggi, Phys. Rep. 304, 229 (1998).

[7] G. Hur, C. E. Creffield, P.H. Jones, and T.S. Monteiro, Phys. Rev. A 72, 013403 (2005).

[8] A. Eckardt, C. Weiss, and M. Holthaus, Phys. Rev. Lett. 95, 260404 (2005).

[9] H. Sambe, Phys. Rev. A 7, 2203 (1973).

[10] M. Holthaus, Z. Phys. B 89, 251 (1992).

[11] C.E. Creffield and G. Platero, Phys. Rev. B 65, 113304 (2002); 66, 235303 (2002).

[12] A. Eckardt, T. Jinasundera, C. Weiss, and M. Holthaus, Phys. Rev. Lett. 95, 200401 (2005).

[13] M. M. Dignam and C. M. de Sterke, Phys. Rev. Lett. 88, 046806 (2002). 